\title{
Diagnostic value of spectral reconstructions in detecting incidental skeletal muscle metastases in CT staging examinations
}

Simon Lennartz ${ }^{1,3}$, Nils Große Hokamp ${ }^{1,2}$, Nuran Abdullayev ${ }^{1}$, Markus Le Blanc ${ }^{1}$, Andra-lza luga', Grischa Bratke ${ }^{1}$, David Zopfs ${ }^{1}$, David Maintz ${ }^{1}$, Jan Borggrefe ${ }^{1}$ and Thorsten Persigehl ${ }^{1 *}$

\begin{abstract}
Background: To investigate if iodine density overlay maps (IDO) and virtual monoenergetic images at $40 \mathrm{keV}$ $\left(V_{M} 4_{40 \mathrm{keV}}\right)$ acquired from spectral detector computed tomography (SDCT) can improve detection of incidental skeletal muscle metastases in whole-body CT staging examinations compared to conventional images.
\end{abstract}

Methods: In total, 40 consecutive cancer patients who underwent clinically-indicated, contrast-enhanced, oncologic staging SDCT were included at this retrospective study: 16 patients with $n=108$ skeletal muscle metastases confirmed by prior or follow-up CT, ${ }^{18}$ F-FDG-PET, MRI or histopathology, and a control group of 24 patients without metastases. Four independent readers performed blinded, randomized visual detection of skeletal muscle metastases in conventional images, IDO and $\mathrm{VMI}_{40 \mathrm{keV}}$, indicating diagnostic certainty for each lesion on a 5-point Likert scale. Quantitatively, ROI-based measurements of attenuation (HU) in conventional images and VMI $\mathrm{V}_{4 \mathrm{kev}}$ and iodine concentration in IDO were conducted. CNR was calculated and receiver operating characteristics (ROC) analysis of quantitative parameters was performed.

Results: Regarding subjective assessment, IDO (63.2 (58.5-67.8) \%) and $\mathrm{VMI}_{40 \mathrm{keV}}(54.4$ (49.6-59.2) \%) showed an increased sensitivity for skeletal muscle metastases compared to conventional images (39.8 (35.2-44.6) \%). Specificity was comparable in $\mathrm{VMI}_{40 \mathrm{kev}}(69.8(63.2-75.8) \%)$ and conventional images (69.2 (60.6-76.9) \%), while in IDO, it was moderately increased to $74.2(65.3-78.4) \%$. Quantitative image analysis revealed that CNR of skeletal muscle metastases to circumjacent muscle was more than doubled in $\mathrm{VMl}_{40 \mathrm{keV}}(25.8 \pm 11.1)$ compared to conventional images $(10.0 \pm 5.3, p \leq 0.001)$. lodine concentration obtained from IDO and $\mathrm{HU}$ acquired from $\mathrm{VMI}_{40 \mathrm{kev}}$ ( $A \cup C=0.98$ each) were superior to $\mathrm{HU}$ attenuation in conventional images ( $\mathrm{UUC}=0.94$ ) regarding differentiation between healthy and metastatic muscular tissue ( $p \leq 0.05)$.

Conclusions: IDO and $\mathrm{VMI}_{40 \mathrm{keV}}$ provided by SDCT improve diagnostic accuracy in the assessment of incidental skeletal muscle metastases compared to conventional $\mathrm{CT}$.

Keywords: Oncologic imaging, Dual energy computed tomography, Skeletal muscle metastases, lodine quantification, lodine maps

\footnotetext{
* Correspondence: thorsten.persigehl@uk-koeln.de

${ }^{1}$ Department of Diagnostic and Interventional Radiology, University of

Cologne, Faculty of Medicine and University Hospital Cologne, Kerpener Str.

62, 50937 Cologne, Germany

Full list of author information is available at the end of the article
}

(c) The Author(s). 2019 Open Access This article is distributed under the terms of the Creative Commons Attribution 4.0 International License (http://creativecommons.org/licenses/by/4.0/), which permits unrestricted use, distribution, and reproduction in any medium, provided you give appropriate credit to the original author(s) and the source, provide a link to the Creative Commons license, and indicate if changes were made. The Creative Commons Public Domain Dedication waiver (http://creativecommons.org/publicdomain/zero/1.0/) applies to the data made available in this article, unless otherwise stated. 


\section{Background}

Soft-tissue metastases of the skeletal muscle (SMM) are less frequent in comparison to liver, lung, bone, or brain metastases [1-8] indicating a high resistance of muscular tissue to metastatic infestation [9]. However, some malignancies, such as melanoma, gastrointestinal, renal and lung cancer, are associated with a relatively high prevalence of SMM [10]. Detection of these lesions is of high clinical interest, as manifestation of SMM is associated with a worsened disease outcome [11] and might switch the treatment to a palliative approach. Although contrast-enhanced CT is the method of choice for fullbody scans in oncologic imaging, it commonly underestimates the prevalence of those particular metastases compared to full-body MRI or PET-CT $[12,13]$. It has been shown recently that iodine density overlay maps (IDO) from dual-energy computed tomography (DECT) have the potential for an improved detection of different metastatic conditions including SMM in melanoma patients [14]. In DECT, one high- and one low-energy dataset is acquired from the polyenergetic $\mathrm{x}$-ray spectrum. This can be achieved through different technical approaches: tube-based DECT scanner work with 1) two sources at different tube voltage (dual-source), 2) two sequent rotations at different tube potentials (dual spin), 3) a technique, in which a beam filter is used to split up an x-ray emitted by a single source in two different-energetic partial beams (twin beam) or 4) rapid switching of tube potentials ( $\mathrm{kVp}$ switching) [15]. In contrast to these source-based concepts, the spectral detector CT (SDCT, Philips Healthcare, Netherlands) operates based on a single-source system working with a dual-layer-detector which registers lower-energetic photons within the upper yttrium-based layer while higher-energetic photons are detected within the lower gadolinium-oxysulphide layer [16]. That approach features concise spatial and angular matching, holding the potential to significantly reduce image noise. Low noise is of special relevance for low $\mathrm{keV}$ virtual monoenergetic images (VMI) which have been shown to improve lesion detection of different metastatic conditions, such as pulmonary or hepatic metastasis but are impaired by an increase in image noise at source-based DECT $[14,17,18]$. Another inherent gain of the detector-based approach is that post-processing takes place within the raw data domain while tube-based DECT either requires previous angular/temporal interpolation or only enables post-processing within the projection domain.

The purpose of this study is to investigate whether VMI at $40 \mathrm{keV}$ and IDO obtained with SDCT can improve both detection rate and diagnostic certainty in the assessment of incidental SMM in patients suffering from various malignant primary diseases.

\section{Methods}

\section{Study cohort}

For this retrospective study, institutional review board approval was obtained. A total of 24 patients with known metastatic disease of the skeletal muscle who received clinically indicated, oncologic SDCT staging examinations in the period from June 2016 to July 2017 were identified. For study inclusion, confirmation either by PET-CT/MRI or previous/follow-up CT with progression in size or change during treatment was mandatory (see below: "lesion annotation"). Out of those 24 initially identified patients, 8 patients had to be excluded because of a) unenhanced CT scan due to known allergic reaction to iodinated contrast agent (1 patient), b) hip/spine-implants causing severe metal artifacts that hampered diagnostic assessment of the circumjacent muscle (4 patients), or c) SMM with a long axis diameter $(\mathrm{LAD})>5 \mathrm{~cm}$ ( 3 patients). The remaining 16 patients were included in the SMM group (group A). As negative controls, 24 patients without SMM who received contrast-enhanced SDCT staging examinations in the same date range as group A were included as a control group (group B). Due the known correlation of extent of metastatic disease and prevalence of SMM, patients in group B were chosen to match cancer stages as of group A in order to minimize confounding effects in the blinded, randomized subjective assessment.

\section{Image acquisition and post-processing}

All patients were scanned on a dual layer detector DECT system (IQon, Philips Healthcare, Best, The Netherlands). The clinical scans were performed in cranio-caudal direction while patients remained in a supine position during inspirational breath-hold.

Our institution's monophasic protocol for CT staging examinations contains a portal venous scan of the chest and abdomen triggered by bolus-tracking technique (threshold of $100 \mathrm{HU}$ in the abdominal aorta) with a delay of $70 \mathrm{~s}$. Administration of $120 \mathrm{ml}$ non-ionic, iodinated contrast media bolus (Accupaque $350 \mathrm{mg} / \mathrm{ml}$, GE Healthcare; Little Chalfort, UK) followed by $30 \mathrm{ml}$ saline chaser is routinely performed with an automated injection system at a flow rate of $3.5 \mathrm{ml} / \mathrm{s}$ (MEDRAD ${ }^{\circ}$ Stellant ${ }^{\oplus}$, Bayer AG, Leverkusen, Germany). The following, constant scan parameters were applied: collimation $-2 \times 64 \times 0.625 \mathrm{~mm}$; rotation time $-0.5 \mathrm{~s}$; pitch - 0.671 ; tube current $-120 \mathrm{kVp}$, matrix $-512 \times 512$; dose modulation was enabled in all patients (DoseRight 3D-DOM, Philips Healthcare, Best, The Netherlands). Mean CTDIvol was $9.0 \mathrm{mGy}$. All images were reconstructed with a slice thickness of $2 \mathrm{~mm}$ and a $1 \mathrm{~mm}$ section increment. Reconstruction of conventional images (CI), VMI and IDO as well as quantitative and qualitative image analysis were performed on a dedicated vendor console 
(IntelliSpace Portal 9.0, Philips Healthcare, Best, The Netherlands). All spectral results were reconstructed with a dedicated, hybrid iterative spectral reconstruction algorithm (Spectral, Kernel B, level 3). As in clinical routine, conventional images were reconstructed using iDose 4 (level 3). To generate IDO, iodine maps were extracted from the spectral data and fused with CI at 120 $\mathrm{kV}$. Iodine maps are material-specific images calculated from the two different-energetic datasets in which the voxel values represent the iodine concentration of the displayed tissue in $\mathrm{mg} / \mathrm{ml}$. Voxels without detectable iodine are equalized to $0 \mathrm{mg} / \mathrm{ml}$ and appear black, accordingly. When being fused with the regular greyscale $\mathrm{CI}$, the iodine concentration in IDO appears color-coded as indicated in Fig. 1.

\section{Lesion annotation}

After study inclusion, SDCT scans of the 16 patients with known metastatic disease of the skeletal muscle were evaluated by two radiologists experienced in oncologic imaging who were not involved in the subsequent subjective image analysis. In a consensus, all metastatic lesions of the muscle were identified, and their slice position was recorded. For that, $\mathrm{CI}, \mathrm{VMI}$ at $40 \mathrm{keV}\left(\mathrm{VMI}_{40 \mathrm{keV}}\right)$ and iodine density overlay maps (IDO) were used and any suspicious lesion was correlated with the aforementioned reference standard (PET-CT/MRI) and/or previous/follow-up CT scans to confirm lesion dignity reliably. In detail, SMM were defined by the following criteria: a) PET-CT-correlation with clearly enhanced nuclide uptake which was classified malignant by a specialist in nuclear medicine and/or

b) MRI-correlation with typical morphology and/or

c) Progression in size without treatment and/or

d) Progression/regression in size during treatment

\section{Subjective analysis}

To determine diagnostic accuracy and certainty in the assessment of SMM, four blinded radiologists with 4-6 years of experience in oncologic imaging independently reviewed $\mathrm{CI}, \mathrm{VMI}_{40 \mathrm{keV}}$ and IDO. For that purpose, the series were randomized and split up to three separate sessions of 40 series each. It was ensured that the same patient did not appear twice within the same session. A latency period of at least four weeks was kept before proceeding with the subsequent session. Within the sessions, the readers were asked to report each lesion suspicious for SMM and name the exact slice position. Additionally, diagnostic certainty regarding lesion dignity was indicated using the following 5-point Likert scale: $1=$ most certainly not a metastasis, $2=$ likely not a metastasis, $3=$ probably a metastasis, $4=$ most likely a metastasis, 5 = definitely a metastasis. Standard window settings of IDO were W/L 3/5 for the iodine density map and W/L 60/360 for CI. To maintain a comparable visual perception in of $\mathrm{CI}$ and $\mathrm{VMI}_{40 \mathrm{keV}}, \mathrm{W} / \mathrm{L}$ were chosen as 690/240 for the latter; however the readers were free to adjust window settings freely as it is wellknown that appropriate window settings are crucial for
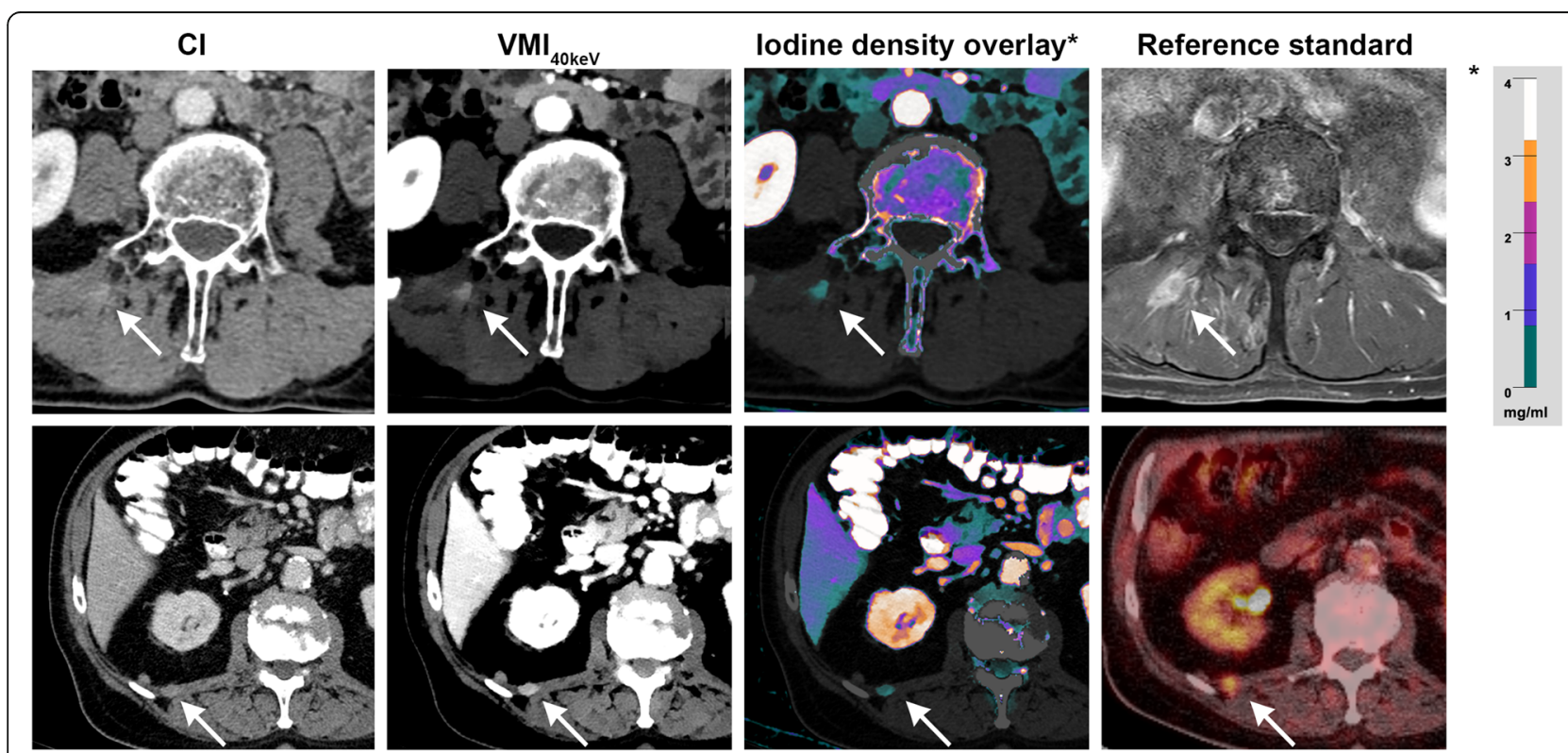

Fig. 1 Exemplary cases of skeletal muscle metastases in $\mathrm{Cl}, \mathrm{VMI}_{40 \mathrm{keV}}$ and IDO with correlating MRI/PET-CT. Upper row: Metastasis of the right autochthone back muscle in a patient with malignant melanoma. Lower row: Patient with lung cancer diagnosed with a metastasis of the right quadratus lumborum muscle. Asterix indicates the color scale used in IDO 
spectral reconstructions $[19,20]$. No time limit for diagnostic assessment was determined.

\section{Quantitative image analysis}

For quantitative image analysis, circular regions of interest (ROIs) were placed within the SMM lesions so that the largest possible area was covered at the slice of the maximum lesion diameter. ROIs within the skeletal muscle and subcutaneous fat were chosen to measure $50 \pm 10 \mathrm{~mm}^{2}$. After initial placement in CI, ROIs were copied and pasted to VMI and IDO to ensure consistent size and localization. The standard deviation within homogeneous fatty tissue was considered to be representative for image noise.

\section{Statistical methods}

Non-parametric Steel-Dwass test (all pairs) was performed to compare quantitative values (HU, IC, CNR) and subjective Likert scores. Sensitivity, specificity, negative, and positive predictive value (NPV/PPV) of the visual assessment were calculated using a contingency table. Inter-rater agreement was determined using Cohen's Kappa which was interpreted as suggested in earlier studies: excellent agreement $(\kappa \geq 0.8)$, good agreement $(\kappa \geq 0.6)$, moderate agreement $(\kappa \geq 0.4)$ and poor agreement $(\kappa \leq 0.4)[21,22]$. Receiver operating characteristics (ROC) analysis of $\mathrm{HU}\left(\mathrm{CI} / \mathrm{VMI}_{40 \mathrm{keV}}\right)$ and $\mathrm{IC}$ was conducted, and Youden's J was calculated to determine optimal cut-off values. Delong method was used to compare different ROC curves [23]. Statistical significance was determined as $p \leq 0.05$. Continuous variables are reported as mean \pm standard deviation (SD) and Likert scores as median and range. Sensitivities and specificities are given with referring 95\% confidence intervals. For statistical analysis, Microsoft Excel (Microsoft Corporation, Redmond, USA), JMP 11 Software (SAS, Cary, USA) and MedCalc 18.2 (Medcalc Software, Ostend, Belgium) were used.

\section{Results}

\section{Study cohort}

SMM group consisted of 16 patients (6 women, 10 men, mean age: $64 \pm 12.9$ years), control group comprised 24 patients (9 women, 15 men, mean age: $66 \pm 12.9$ years), showing similar age distribution $(p=0.87)$. Underlying diseases (including melanoma, esophageal cancer, renal cancer, lung cancer, breast cancer, neuroendocrine tumor, and sarcoma) are shown in Table 1.

\section{Subjective analysis}

Subjective analysis revealed a higher sensitivity for SMM in IDO (63.2 (58.5-67.8) \%) as well as in $\mathrm{VMI}_{40 \mathrm{keV}}(54.4$ (49.6-59.2) \%) compared to CI (39.8 (35.2-44.6) \%). Overall specificity was marginally higher in IDO (74.2
Table 1 Distribution of underlying diseases in patients with skeletal muscle metastases (SMM) and control group patients

\begin{tabular}{lll}
\hline Underlying disease & SMM group & Control group \\
\hline Melanoma & 7 & 3 \\
Esophageal carcinoma & 3 & 4 \\
Pancreatic cancer & 0 & 5 \\
Breast cancer & 1 & 3 \\
Lung cancer & 1 & 1 \\
Renal cell carcinoma & 2 & 0 \\
Sarcoma & 1 & 2 \\
Neuroendocrine tumor & 1 & 2 \\
Gastrointestinal stroma tumor & 0 & 1 \\
Colorectal cancer & 0 & 1 \\
Cholangiocellular carcinoma & 0 & 1 \\
Urothelial carcinoma & 0 & 1 \\
\hline
\end{tabular}

(65.3-78.4) \%) compared to $69.2(60.6-76.9) \%$ in $\mathrm{CI}$ and $69.8(63.2-75.8) \%$ in $\mathrm{VMI}_{40 \mathrm{keV}}$. Although indicating a tendency towards better detection of hypervascularized SMM lesions, the influence of iodine concentration on sensitivity was limited: in IDO, sensitivity for lesions with an iodine uptake within the upper two quartiles [1.69-4.32 $\mathrm{mg} / \mathrm{ml}]$ was $65.5(58.8-71.7) \%$, while for the lower two quartiles [0.0-1.69 mg/ml], it was $61.1(54.3-$ $67.7) \%$. Diagnostic certainty for correctly identified SMM tended to be superior in $\mathrm{VMI}_{40 \mathrm{keV}}$ (median 5) compared to CI and IDO (median 4) which was not statistically significant (p-range: 0.16-0.8) (Fig. 2). Inter-rater agreement in identifying patients with SMM was good in CI $(\kappa=0.62)$ and IDO $(\kappa=0.76)$ and moderate in $\mathrm{VMI}_{40 \mathrm{keV}}(\mathrm{K}=0.44)$. Detailed results of the subjective assessment for every individual reader are shown in Table 2.

\section{Quantitative analysis}

Attenuation in CI (lesion: 93.4 \pm 26.7 , muscle: $45.4 \pm$ 23.3) and $\mathrm{VMI}_{40 \mathrm{keV}}$ (lesion: $194.5 \pm 67.9$, muscle:50.6 \pm 32.7) as well as iodine concentration (lesion: $1.8 \pm 0.8$ $\mathrm{mg} / \mathrm{ml}$, muscle: $0.3 \pm 0.2 \mathrm{mg} / \mathrm{ml}$ ) was significantly higher in SMM compared to circumjacent skeletal muscle $(p \leq 0.001)$. CNR of SMM to circumjacent muscle was significantly increased compared to CI (10.1 \pm $5.3)$ in VMI ranging from 40 to $60 \mathrm{keV}(p \leq 0.001)$ with a maximum CNR of $25.8 \pm 11.1$ in $\mathrm{VMI}_{40 \mathrm{keV}}(p \leq 0.001)$. VMI at $70 \mathrm{keV}$ yielded an CNR equivalent to CI (10.5 \pm 5.3; $p=0.3$ ), while in VMI from 80 to $200 \mathrm{keV} \mathrm{CNR} \mathrm{was}$ significantly lower $(p \leq 0.05)$. ROC analysis yielded a superior differentiation of SMM and healthy muscle in $\mathrm{VMI}_{40 \mathrm{keV}}$ and IDO (AUC $=0.98$ each) compared to $\mathrm{CI}$ (AUC $=0.94, p \leq 0.05$ ). Youden's indices revealed optimal cut offs of $>62.1 \mathrm{HU}$ for $\mathrm{CI},>107.7 \mathrm{HU}$ for $\mathrm{VMI}_{40 \mathrm{keV}}$ and $>0.73 \mathrm{mg} / \mathrm{ml}$ for IDO. 4 out of 108 included lesions 


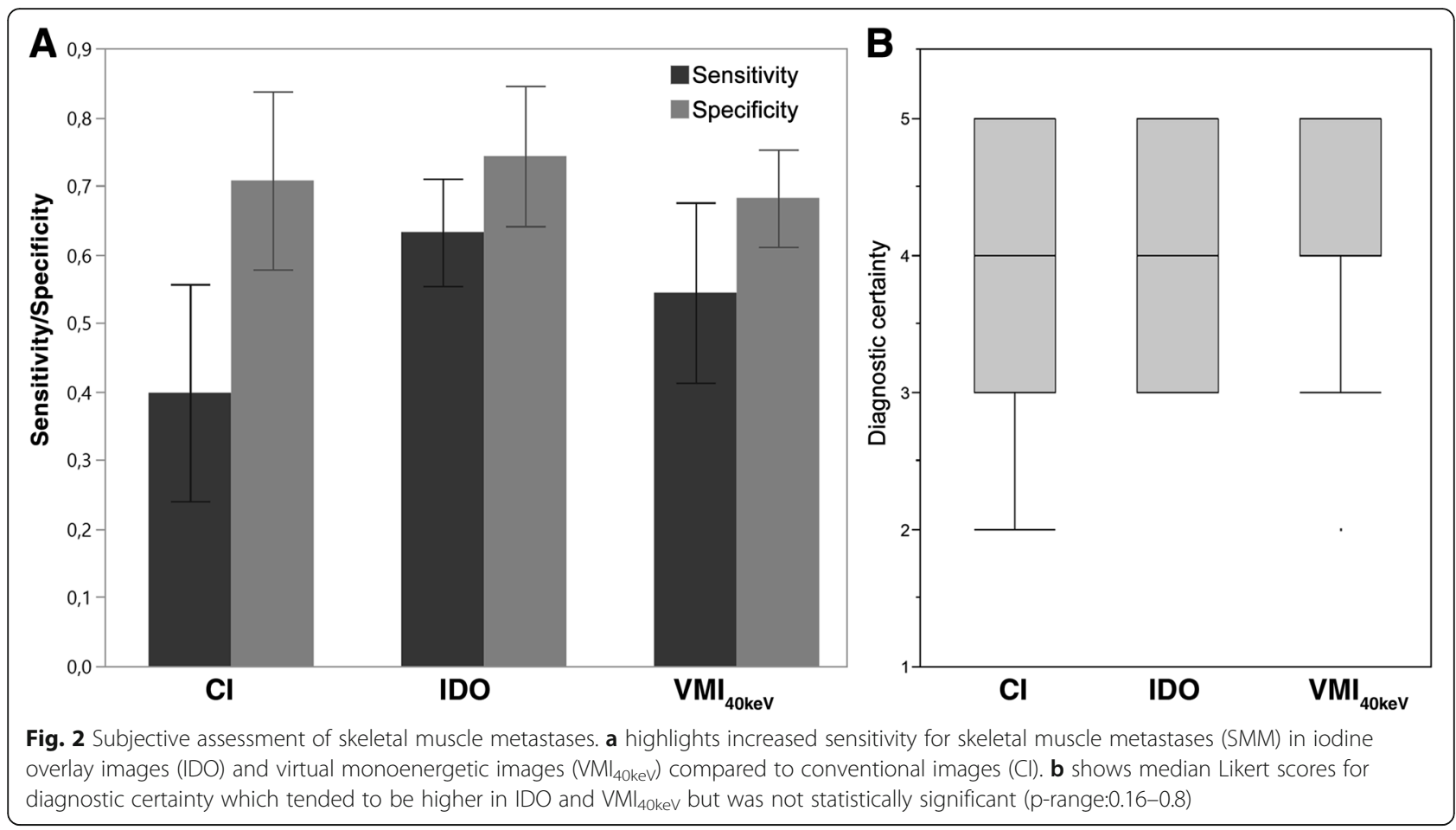

(3.7\%) showed necrosis with corresponding decrease in mean iodine uptake to $0.5 \pm 0.4 \mathrm{mg} / \mathrm{ml}$ compared to $1.8 \pm$ $0.8 \mathrm{mg} / \mathrm{ml}$ in non-necrotic lesions $(p \leq 0.05$; Fig. $3 \mathrm{f})$. Mean long axis diameter of SMM lesions was $1.1 \pm 0.6$ $\mathrm{cm}$. Distribution of lesion size is shown in Fig. 3f.

\section{Discussion}

This study investigates if and to what extent iodine density overlay maps (IDO) and virtual monoenergetic images obtained with spectral detector computed tomography (SDCT) can improve visual and quantitative diagnostic accuracy in assessing incidental skeletal muscle metastases (SMM). In CT, the assessment of the muscular system regarding metastatic lesions is severely hampered by different factors: the skeletal muscles constitute a large volume to investigate and usually show high attenuation so that iso- and moderately hyperattenuating metastases may get obscured. In the past, dual energy CT (DECT)-derived reconstructions highlighting the iodine signal have been shown to increase contrast and improve depiction of malignant lesions such as liver metastases, hepatocellular carcinoma, pancreatic or lung tumors [24-29]. This can be achieved using iodine-specific maps or lower-energetic virtual monochromatic images which result in a higher $\mathrm{x}$-ray absorption of iodine as the energy level is moved closer to its absorption maximum, the k-edge (33.2 keV) [30].

Our study revealed that VMI at $40 \mathrm{keV}\left(\mathrm{VMI}_{40 \mathrm{keV}}\right)$ allowed for a significantly improved contrast of SMM in comparison to the circumjacent tissue resulting in a moderately increased detection rate of those lesions. These results are in line with earlier studies that described improved detection of hyperattenuating lesions in lowenergy virtual monoenergetic images [26]. However, in

Table 2 Diagnostic accuracy in the detection of skeletal muscle metastases in conventional images (CI), iodine overlay images (IDO) and virtual monoenergetic images at $40 \mathrm{keV}\left(\mathrm{VMI}_{40 \mathrm{keV}}\right)$

\begin{tabular}{|c|c|c|c|}
\hline \multirow[t]{2}{*}{ READER } & \multicolumn{3}{|l|}{ Sensitivity/Specificity (\%) } \\
\hline & $\mathrm{Cl}$ & IDO & $\mathrm{VMI}_{40 \mathrm{keV}}$ \\
\hline 1 & $35.2(26.2-45.0) / 75.0(53.3-90.2)$ & $56.5(46.6-66) / 84.2(60.4-96.6)$ & $48.1(38.4-58.0) / 63.6(40.7-82.8)$ \\
\hline 2 & $57.4(47.5-66.9) / 59.1(43.4-73.7)$ & $73.1(63.8-81.2) / 78.8(67.0-87.9)$ & $64.8 /(55.0-73.8) 74.7(65.0-82.9)$ \\
\hline 3 & $46.3(36.7-56.2) / 61.8(43.6-77.8)$ & $65.7(56.0-74.6) / 60.3(47.2-72.4)$ & 65.7 (56.0-74.6) / 60.7 (46.8-73.5) \\
\hline 4 & $20.4(13.2-29.2) / 87.1$ (70.2-96.4) & $57.4(47.5-66.9) / 73.9(58.9-85.7)$ & 38.9 (29.7-48.8) / 73.7 (56.9-86.6) \\
\hline OVERALL & 39.8 (35.2-44.6) / $69.2(60.6-76.9)$ & $63.2(58.5-67.8) / 74.2(65.3-78.4)$ & $54.4(49.6-59.2) / 69.8(63.2-75.8)$ \\
\hline Cohen's Kappa & $K=0.62$ & $K=0.76$ & $K=0.44$ \\
\hline
\end{tabular}




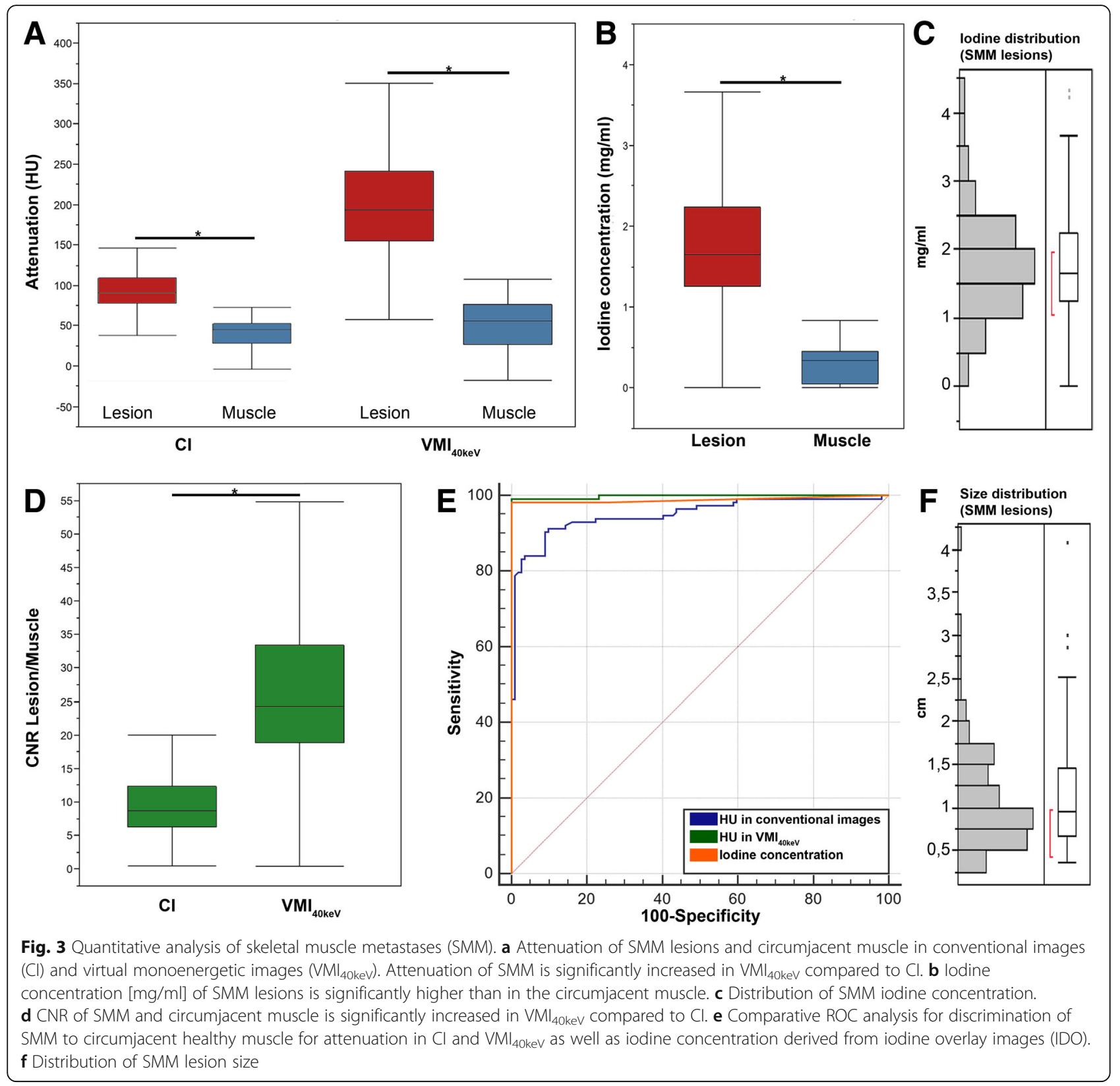

iodine density overlay maps (IDO) in which the iodine signal is color-coded and merged with conventional images (CI), the observed boost in sensitivity compared to $\mathrm{CI}$ was markedly higher than in $\mathrm{VMI}_{40 \mathrm{keV}}$. On the other hand, specificity was only slightly higher in IDO and comparable between $\mathrm{VMI}_{40 \mathrm{keV}}$ and CI. A recent study by Uhrig et al. investigated subjective assessment of different distant metastases in patients with melanoma using iodine-overlay images derived from a dual-source CT scanner including a minor subgroup of 13 SMM lesions [14]. They reported a sensitivity for SMM of $8 \%$ in conventional images which was boosted up to $99 \%$ in iodine overlay images. Compared to these results, overall sensitivity in CI observed in our study was much higher (39.8 (35.2-44.6) \%) while in IDO it was lower (63.2 (58.5-67.8) \%) which might be explicable by the very small sample size of the SMM subgroup Uhrig et al. examined. In our study, inter-rater agreement for the subjective assessment of SMM was good in $\mathrm{CI}(\mathrm{K}=0.62)$ and IDO $(\mathrm{\kappa}=0.76)$ and moderate in $\mathrm{VMI}_{40 \mathrm{keV}}(\mathrm{K}=0.44)$. The observed decrease in inter-rater agreement and specificity in $\mathrm{VMI}_{40 \mathrm{keV}}$ might be owed to the fact that iodine contrast boost may have led to misinterpretation of inhomogeneous muscular contrast enhancement in some cases resulting in false positives. The subjective results are in line with the quantitative image analysis which revealed $\mathrm{HU}$ acquired from 
$\mathrm{VMI}_{40 \mathrm{keV}}$ and iodine concentration obtained from IDO to be superior to conventional $\mathrm{HU}$ in differentiating metastatic lesions from circumjacent, unaffected muscle.

Given the results of the subjective assessment, IDO seem the most predestined spectral reconstruction to screen for incidental metastatic muscle infiltration in regular, clinically-indicated CT staging examinations of the chest, abdomen and pelvis. Consequently, they have been implemented into the clinical workflow at our institute for patients suffering from diseases with increased pre-test probability for SMM: melanoma, gastrointestinal tumors, renal cancer, lung cancer, thyroid gland carcinoma, breast cancer, and carcinoma of unknown primary (CUP) [10]. Still, it needs to be stated that the sensitivity of $63 \%$ yielded by IDO, although being superior to conventional CT images, is still suboptimal for oncologic imaging purposes. Thus, for some indications, such as staging of metastatic melanoma, ${ }^{18}$ F-FDG PET/ $\mathrm{CT}$ reflects the best imaging method. However, in staging examinations for patients who do not qualify for regular oncologic follow-up with ${ }^{18} \mathrm{~F}$-FDG PET/CT, such as patients with metastatic renal cancer, IDO may help to detect incidental SMM.

Aside from the retrospective nature of our study, there are various limitations: First, the number of 16 included patients with SMM is relatively small. However, considering the number of 108 included lesion and the blinded, randomized study design with 4 independent subjective readers, significance of results still seems sufficiently powered. Overall, the number of eligible patients for inclusion was limited due to the overall low prevalence of metastatic disease of the skeletal muscle as well as the required reference standard necessary for study inclusion. Due to the small patient number there is the possibility of recognition bias which may have influenced the diagnostic accuracy. Another factor that may play a role in this context is possible localization of lesions at memorable sites such as extremities. Recognition effects were minimized as far as possible by a large time frame of at least 4 weeks and randomization of patient/series between repetitive subjective examinations. Still, blinding to reconstructed series was not possible as CI, $\mathrm{VMI}_{40 \mathrm{keV}}$ and IDO can be easily recognized due to their characteristic visual impression. Secondly, it is known that individual intravenous iodine load varies between different scans dependent on circulation and other physiological and technical factors which might potentially influence the iodine density of SMM lesions. Nonetheless, unlike previous studies on DECT-derived iodine concentration, normalization to intravenous iodine load was waived in our study as SMM were allocated within the complete thoracoabdominal scan volume so that normalization to a central vessel e.g. abdominal aorta did not seem to be representative. Lastly, the majority of included SMM lesions were iso- or hyperattenuating in relation to circumjacent skeletal muscle; in fact, only 4 out of 108 included lesions showed central necrosis. This relatively small proportion of necrotic lesions might be on account of the initial exclusion of lesions with a long axis diameter $>5 \mathrm{~cm}$. As large lesions tend to show necrosis more frequently, the study cohort might be biased in terms of these necrotic lesions. However, large SMM might be also easily depicted without the use of spectral reconstructions. As a limitation due to the relatively small sample size, it was not possible to statistically determine if there was a correlation between lesion necrosis and detection rate.

\section{Conclusions}

Iodine overlay images obtained with spectral detector CT improve visual and quantitative diagnostic accuracy in assessing skeletal muscle metastases (SMM) compared to conventional images. Hence, these reconstructions seem advantageous at regular oncological CT examinations regarding the detection of incidental SMM particularly for patients suffering from metastatic malignancies and yet not qualifying for routine ${ }^{18}$ F-FDG PET/ CT imaging.

\section{Abbreviations \\ Cl: Conventional images; DECT: Dual-energy computed tomography; IC: Iodine concentration; IDO: lodine density overlay maps; SDCT: Spectral detector computed tomography; SMM: Skeletal muscle metastases; $\mathrm{VMI}_{40 \mathrm{keV}}$ : Virtual monoenergetic images at $40 \mathrm{keV}$}

\section{Acknowledgements}

Not applicable.

\section{Authors' contributions}

$\mathrm{NA}, \mathrm{ML}$, All, GB conducted the subjective analysis. SL and JB performed lesion annotation and quantitative analysis. SL drafted the manuscript and performed statistical analyses. TP and JB gave significant contribution to study design. TP, NGH, DM, DZ contributed to manuscript preparation and revision. NGH substantially contributed to the statistical analysis. All authors read and approved the final manuscript.

\section{Funding}

No funding to declare for this manuscript.

Availability of data and materials

Please contact the corresponding author for data requests.

\section{Ethics approval and consent to participate}

This study was approved by the Institutional Review Board and informed consent was waived due to the retrospective character of the study.

\section{Consent for publication}

Not applicable.

\section{Competing interests}

$S L$ received research and travel support not related to this specific project from Philips Healthcare. JB, DM, NGH received speaker's honoraria from Philips Healthcare. All other authors: Nothing to disclose.

\section{Author details}

${ }^{1}$ Department of Diagnostic and Interventional Radiology, University of Cologne, Faculty of Medicine and University Hospital Cologne, Kerpener Str. 62, 50937 Cologne, Germany. 'Department of Radiology, Case Western 
Reserve University and University Hospitals, Cleveland, OH, USA. ${ }^{3}$ Else Kröner Forschungskolleg Clonal Evolution in Cancer, University Hospital Cologne, Weyertal 115b, 50931 Cologne, Germany.

Received: 16 January 2019 Accepted: 28 June 2019

Published online: 17 July 2019

\section{References}

1. Hasegawa S, Sakurai Y, Imazu H, Matsubara T, Ochiai M, Funabiki T, et al. Metastasis to the forearm skeletal muscle from an adenocarcinoma of the Colon: report of a case. Surg Today. 30:1118-23. https://doi.org/10.1007/ s005950070013

2. Marioni G, Blandamura S, Calgaro N, Ferraro SM, Stramare R, Staffieri A, Filippis CD. Distant muscular (gluteus maximus muscle) metastasis from laryngeal squamous cell carcinoma. Acta Otolaryngol. 2005;125:678-82. https://doi.org/10.1080/00016480410024613

3. CM P. Incidence and type of pathologic alterations observed in muscle in a routine autopsy survey. Neurology. 1959;9:757-66.

4. Sridhar KS, Rao RK, Kunhardt B. Skeletal muscle metastases from lung cancer. Cancer. 1987:59:1530-4.

5. Mulsow FW. Metastatic carcinoma of skeletal muscles. Arch Pathol. 1943:112-4.

6. Chandler RW, Shulman I, Moore TM. Renal cell carcinoma presenting as a skeletal muscle mass: A case report. Clin Orthop Relat Res. 1979:227-9.

7. Laurence AE, Murray AJ. Metastasis in skeletal muscle secondary to carcinoma of the colon--presentation of two cases. Br J Surg. 1970;57:529-30

8. Perisano C, Spinelli MS, Graci C, Scaramuzzo L, Marzetti E, Barone C, et al. Soft tissue metastases in lung cancer: a review of the literature. Eur Rev Med Pharmacol Sci. 2012;16:1908-14.

9. Bar-Yehuda S, Barer F, Volisson L, Fishman P. Resistance of muscle to tumor metastases: a role for A3 adenosine receptor agonists. Neoplasia. 2001;3:125-31. https://doi.org/10.1038/sj.neo.7900138

10. Surov A, Hainz M, Holzhausen H-J, Arnold D, Katzer M, Schmidt J, et al. Skeletal muscle metastases: primary tumours, prevalence, and radiological features. Eur Radiol. 2010;20:649-58. https://doi.org/10.1007/ s00330-009-1577-1

11. Pop D, Nadeemy AS, Venissac N, Guiraudet P, Otto J, Poudenx M, Mouroux J. Skeletal muscle metastasis from non-small cell lung cancer. J Thorac Oncol. 2009;4:1236-41. https://doi.org/10.1097/JTO.0b013e3181 b24509

12. Nguyen NC, Chaar BT, Osman MM. Prevalence and patterns of soft tissue metastasis: detection with true whole-body F-18 FDG PET/CT. BMC Med Imaging. 2007;7:8. https://doi.org/10.1186/1471-2342-7-8

13. Wu CC, Khorashadi L, Abbott GF, Gilman MD. Common blind spots on chest CT: where are they all hiding? Part 1-airways, lungs, and pleura. AJR Am J Roentgenol. 2013;201:W533-8. https://doi.org/10.2214/AJR.12. 9354

14. Uhrig M, Simons D, Bonekamp D, Schlemmer H-P. Improved detection of melanoma metastases by iodine maps from dual energy CT. Eur J Radiol. 2017;90:27-33. https://doi.org/10.1016/j.ejrad.2017.02.024

15. Silva AC, Morse BG, Hara AK, Paden RG, Hongo N, Pavlicek W. Dual-energy (spectral) CT: applications in abdominal imaging. Radiographics. 2011;31: 1031-46; discussion 1047-50. https://doi.org/10.1148/rg.314105159

16. McCollough CH, Leng S, Yu L, Fletcher JG. Dual- and multi-energy CT: principles, technical approaches, and clinical applications. Radiology. 2015;276:637-53. https://doi.org/10.1148/radiol.2015142631

17. Yu L, Leng S, McCollough CH. Dual-energy CT-based monochromatic imaging. AJR Am J Roentgenol. 2012;199:S9-S15. https://doi.org/10.2214/ AJR.12.9121

18. Kalender WA, Klotz E, Kostaridou L. An algorithm for noise suppression in dual energy CT material density images. IEEE Trans Med Imaging. 1988;7:218-24. https://doi.org/10.1109/42.7785

19. Hickethier T, luga A-I, Lennartz S, Hauger M, Byrtus J, Luetkens JA, et al. Virtual Monoenergetic images from a novel dual-layer spectral detector computed tomography scanner in portal venous phase: adjusted window settings depending on assessment focus are essential for image interpretation. J Comput Assist Tomogr. 2018. https://doi.org/10.1097/RCT. 0000000000000711

20. Große Hokamp N, Kessner R, van Hedent S, Graner FP, Gupta A, Gilkeson R. Spectral detector computed tomography pulmonary angiography: improved diagnostic assessment and automated estimation of window settings angiography of pulmonary arteries from novel spectral detector computed tomography provides improved image quality if settings are adjusted. J Comput Assist Tomogr. 2018. https://doi.org/10.1097/RCT. 0000000000000743

21. Fleiss $\mathrm{J}$, Cohen J. The equivalence of weighted kappa and the Intraclass correlation coefficient as measures of reliability. Educ Psychol Meas. 2016;33:613-9. https://doi.org/10.1177/001316447303300309

22. Cohen J. A coefficient of agreement for nominal scales. Educ Psychol Meas. 2016;20:37-46. https://doi.org/10.1177/001316446002000104

23. DeLong ER, DeLong DM, Clarke-Pearson DL. Comparing the areas under two or more correlated receiver operating characteristic curves: a nonparametric approach. Biometrics. 1988;44:837-45.

24. Patel BN, Thomas JV, Lockhart ME, Berland LL, Morgan DE. Single-source dual-energy spectral multidetector CT of pancreatic adenocarcinoma: optimization of energy level viewing significantly increases lesion contrast. Clin Radiol. 2013;68:148-54. https://doi.org/10.1016/j.crad.2012.06.108

25. Agrawal MD, Pinho DF, Kulkarni NM, Hahn PF, Guimaraes AR, Sahani DV. Oncologic applications of dual-energy CT in the abdomen. Radiographics. 2014;34:589-612. https://doi.org/10.1148/rg.343135041

26. Große Hokamp N, Höink AJ, Doerner J, Jordan DW, Pahn G, Persigehl T, et al. Assessment of arterially hyper-enhancing liver lesions using virtual monoenergetic images from spectral detector $\mathrm{CT}$ : phantom and patient experience. Abdom Radiol (NY). 2017. https://doi.org/10.1007/s00261-01 7-1411-1

27. Shuman WP, Green DE, Busey JM, Mitsumori LM, Choi E, Koprowicz KM, Kanal KM. Dual-energy liver CT: effect of monochromatic imaging on lesion detection, conspicuity, and contrast-to-noise ratio of hypervascular lesions on late arterial phase. AJR Am J Roentgenol. 2014;203:601-6. https://doi. org/10.2214/AJR.13.11337

28. Zhang LJ, Yang GF, Wu SY, Xu J, Lu GM, Schoepf UJ. Dual-energy CT imaging of thoracic malignancies. Cancer Imaging. 2013;13:81-91. https:// doi.org/10.1102/1470-7330.2013.0009

29. Shimamoto H, Iwano S, Umakoshi H, Kawaguchi K, Naganawa S. Evaluation of locoregional invasiveness of small-sized non-small cell lung cancers by enhanced dual-energy computed tomography. Cancer Imaging. 2016:16:18. https://doi.org/10.1186/s40644-016-0077-1

30. Riederer SJ, Mistretta CA. Selective iodine imaging using K-edge energies in computerized x-ray tomography. Med Phys. 1977;4:474-81. https://doi.org/1 $0.1118 / 1.594357$

\section{Publisher's Note}

Springer Nature remains neutral with regard to jurisdictional claims in published maps and institutional affiliations.

Ready to submit your research? Choose BMC and benefit from:

- fast, convenient online submission

- thorough peer review by experienced researchers in your field

- rapid publication on acceptance

- support for research data, including large and complex data types

- gold Open Access which fosters wider collaboration and increased citations

- maximum visibility for your research: over $100 \mathrm{M}$ website views per year

At $\mathrm{BMC}$, research is always in progress.

Learn more biomedcentral.com/submission 